\title{
Isolation of Lung Cancer from Inflammation
}

\author{
Md. Jahangir Alam ${ }^{1}$, Sid Ray ${ }^{1}$, and Hiromitsu Hama ${ }^{2}$ \\ 1 Clayton School of Information Technology, Monash University, \\ Victoria 3800, Australia \\ \{md.alam, sid\}@csse.monash.edu.au \\ 2 Faculty of Engineering, Osaka City University, \\ 3-3-138 Sugimoto, Osaka, Japan \\ hama@info.eng.osaka-cu.ac.jp
}

\begin{abstract}
In this paper we propose an efficient new algorithm for making an intelligent system for isolating the lung cancer from the inflamed region using needle biopsies. The best way among the cancer treatments, surgery, is the way that can be used for the removal of a malignant tumor in an operation. It is most effective when a cancer is small and localized. Identification and removal of the cancer cells in their earliest formation are very much important. Almost all of the diagnostic laboratories in the world use experts to identify the suspected cells of the lung tumors under microscope. Due to the smaller number of experts, the proposed method, derived based on image contour analysis, has an important significance to replace the manual methods by an intelligent system.
\end{abstract}

\section{Introduction}

Cancer is a group of diseases characterized by the uncontrolled growth of abnormal cells in the body. Once cells become malignant, they multiply more rapidly than usual. Then they often form masses called tumors that invade nearby tissue and interfere with normal bodily functions. Cancer cells also have a tendency to spread to other parts of the body, where they may form a secondary tumor. The surgical approach, the best way among the existing cancer treatments, is most effective when cancer is small and localized.

Due to the rapid developments of image processing and pattern recognition techniques, computer-aided lung cancer diagnosis has drawn additional attraction. Many accomplishments have already been achieved [1, 2, ,3]. In an effort to improve accuracy and the speed of lung cancer pulmonary radiology, an artificial neural network-based hybrid lung cancer detection method was designed 4]. The false-negative cases in mass screening of lung cancer were analyzed in [5], 6]. Koizumi [7] showed how cancer cell could be separated from the inflammation by analyzing the high and low components of Fourier Descriptors of cell contours. A system that extracted and analyzed features of the lung and pulmonary blood vessel regions for the detection of tumor candidate from helical $\mathrm{CT}$ images is described in 8 .

In the work reported in this paper we use the compactness of the cell contour, in conjunction with the equilibrium number of curvature zero-crossing points, 
to analyze the contour of the suspected cancer cell. The method of compactness analysis is used based on the fact that the cancer cells (type-C1 and type-C2) have spike-wheel contours. Due to the wheel shape, this type of cell is more compact, unless the contour contains high frequencies. On the other hand, the inflmatory cells type-B1 and type-B2 have non-wheel contour without spikes on them. The rest of the paper is organized as follows. Section 2 presents the proposed cancer cell isolation system. Section 3 shows the experimental results and conclusion follows in Section 4 .

\section{Cancer Cell Isolation System}

The typical cancer cell isolation system has four parts: image and contour extraction, estimation of equilibrium number of curvature zero-crossing points, compactness analysis varying scale-space parameter and cancer cell isolation decision. The following three sub-sections describe these components.

\subsection{Image Capture and Contour Extraction}

A digital camera is mounted with the light microscope for capturing the suspected cell images of the specimens of needle biopsies. Four cancer cell countours: type-B1, type-B2, type-C1, and type-C2 are shown in Fig. 1)(a). It is to be noted here that cancer cells type-B1 and type-B2 are not dangerous; these cells form the inflammatory area in the lung region. In this paper, details of image capture, preprocessing and contour extraction are not included. All the cell contours are stored in the database. Each contour is normalized with 360 points implementing interpolation using FFT method 9]. Other interpolation methods are also checked in but FFT method provides the best outcome.

\subsection{Equilibrium Number of Zero-Crossing Points}

The equilibrium number of curvature zero-crossing points is found by varying the scale-space parameter. The scale-space parameter is chosen in such a way that its value is neither too small nor too large so that we can avoid the influence of noise effects and the loss of important features of the contour. When the contour attains the equilibrium number of zero-crossing points, it remains unchanged for a sufficient range of scale-space parameter values.

Of the many approaches to shape representation and analysis that have been proposed, the notion of curvature of planar curves has emerged to be one of the most powerful. There is psychophysical and physiological as well as computational and mathematical support in favor of using curvature as a representation for contours.

Let us suppose $\beta(s)$ is a parameterized contour defined by $\beta(s)=(x(s), y(s))$, $s \in[0,1]$. An evolved version of the contour $\beta_{\sigma}(s)$ can be computed as follows:

$$
\beta_{\sigma}(s)=(x(s, \sigma), y(s, \sigma)), s \in[0,1]
$$




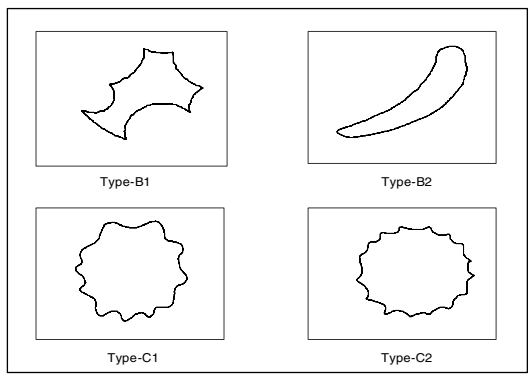

(a)

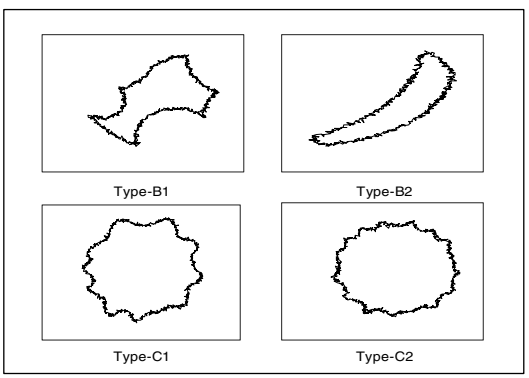

(b)

Fig. 1. Different types of cell contours: (a) database contours and (b) test contours

where $x(s, \sigma)=x(s) \otimes g(s, \sigma), y(s, \sigma)=y(s) \otimes g(s, \sigma), \otimes$ is the convolution operator and $g(s, \sigma)$ denotes a Gaussian kernel of width $\sigma$ :

$$
g(s, \sigma)=\frac{1}{\sigma \sqrt{2 \pi}} e^{\frac{-s^{2}}{2 \sigma^{2}}}
$$

The curvature $K$ on $\beta_{\sigma}$ is given by 10

$$
K(s, \sigma)=\frac{x^{\prime}(s, \sigma) y^{\prime \prime}(s, \sigma)-x^{\prime \prime}(s, \sigma) y^{\prime}(s, \sigma)}{\left(x^{\prime 2}(s, \sigma)+y^{\prime 2}(s, \sigma)\right)^{\frac{3}{2}}}
$$

The curvature zero-crossing points can be found by setting $K(s, \sigma)=0$. There is no way to know a priori what scale to search to smooth the contour. One strategy followed is to search for the position in scale-space where the number of dominant points is stable over the largest range of scales. We select a particular value of $\sigma$ for which the contour of the object attains a certain number of curvature zero-crossing points, which remains unchanged for a long period of its changes. Fig. 2 shows an evolving contour of cancer type-B1 using different levels of scale-space parameter.

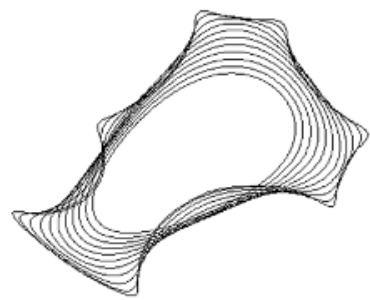

Fig. 2. Evolving contour using different levels of scale-space parameter 


\subsection{Compactness Analysis and Cancer Cell Isolation}

The estimated values of the compactness for the input and database contours at an equilibrium number of zero-crossing points condition are used for the isolation of each cancer cell. As in [9], we measure the compactness of a contour by

$$
\gamma=\frac{(\text { perimeter })^{2}}{4 \pi(\text { area })}
$$

Fig. 3 shows the variation of the compactness of the database (see Fig. 1(a)) and test contours (see Fig. 1(b)) for various levels of the scale-space parameter.

The test contours are identified based on two conditions: equilibrium number of zero-crossing points $\left(N_{e q}\right)$ and the compactness at the equilibrium $\left(\gamma_{e q}\right)$. If the number of zero-crossing points $(N)$ is high $\left(N>N_{e q}\right)$ and the compactness $(\gamma)$ is low $\left(\gamma<\gamma_{e q}\right)$, the cell is malignant. This means that the person whose lung cells have been tested, is very likely to be suffering from lung cancer. The person should go for further investigation to identify the level and the location of the tumor in the lung region. If $N$ is low $\left(N<N_{e q}\right)$ and $\gamma$ is high $\left(\gamma>\gamma_{e q}\right)$, the cell is inflammatory (not dangerous). The conditions, when $\gamma$ is low and $N$ is also low or $\gamma$ is high and $N$ is also high, represent undecided cases.

To find out the values of $N_{e q}$ and $\gamma_{e q}$ we use the findings from our experimental observation. We know that the structure of a malignant cell is spikewheel-shaped. From the investigation of the experimental findings, the values of $N_{e q}$ and $\gamma_{e q}$ have been chosen as 15 and 1.5, respectively. The number of zerocrossing points sustain at the equilibrium level for a certain range of scale-space parameter. The compactness of each contour has been calculated when the equilibrium number of zero-crossing points first appears corresponding to the value of $\sigma$. To find out the equilibrium number of zero-crossing points, we change the value of $\sigma$ sufficiently small in each step.

\section{Experimental Results}

In examining the performance of the proposed method, experiments were conducted on different cancer cells. Twenty sets of test cancer cell contour are constructed using noise and removing a small portion of the contour to show the robustness in the case of contour deformation. Fig. 1(b) shows one set of constructed test contours corresponding to the database contours in Fig. 1(a). The algorithm has been programmed in Matlab and implemented on a Pentium III personal computer.

From Fig. 3it is clear that even if the test contours contain noise, the ultimate variation of the compactness remains in the same level for a sufficient range of scale-space parameter. For the cells type-C1 and type-C2, the compactness value $(N)$ becomes almost 1.0 (contour becomes round shaped) for scale-space parameter $(\sigma)$ values in the range 18-20. At the same level of the scale, the compactness for both cells type-B1 and type-B2 is more than 1.5. Cells type$\mathrm{B} 1$ and type-B2 take far higher range of $\sigma$ values for the contour shape to be elliptical. 


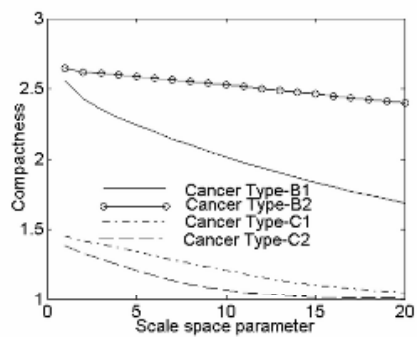

(a)

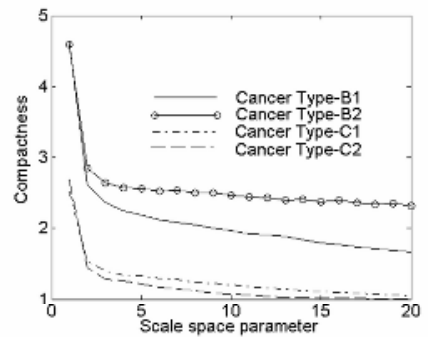

(b)

Fig. 3. Compactness variation for (a) database and (b) test contours

Fig. 4 shows the variation of the number of curvature zero-crossing points for various levels of the scale-space parameter for the database and the test contours. For the test contour of type-B1, the equilibrium number of zero-crossing points becomes 10, whereas for the same type in the database contour this number is 12. For the test contour of cancer cell type-B2, the equilibrium number of zerocrossing points is attained at the value of 2 , which is the same for the database contour. For cancer cell types-C1, the number of zero-crossing points becomes 19 , which is very close to the number of zero-crossing points for the database contour of the type-C1. For the case of cancer cell type-C2, the number of zero-crossing points is 30 , which is almost the same number as in the database contour.

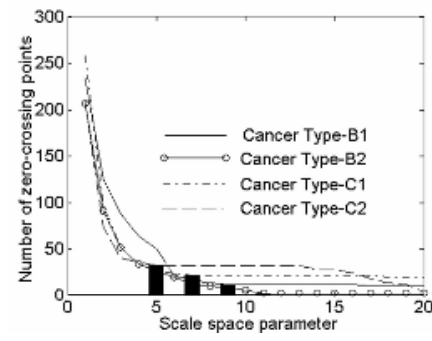

(a)

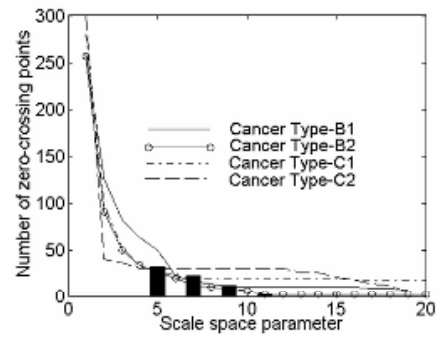

(b)

Fig. 4. Equilibrium zero-crossing points (bar) for (a) database and (b) test contours

\section{Conclusion}

In this research study we have dealt with the problem of cancer cell isolation from inflammation using needle biopsies. From the the compactness analysis at the equilibrium number of curvature zero-crossing points, each test contour is correctly identified. In most cases, the equilibrium number of zero-crossing points for cancer cell contours type-C1 and type-C2, is higher than 15 and the compactness value is less than 1.5. On the other hand, for the non-dangerous 
cells type-B1 and type-B2, the compactness value does not go bellow 1.5 in the equilibrium range of zero-crossing points. Furthermore, the number of zerocrossing points maintains its value under 15 . The proposed method is very simple but effective in its use for the isolation of lung cancer from inflammation. A limitation of this study is that the test cases are made artificially. Our future goal is to implement the proposed system on a set of real data obtained from a sufficient number of suspected lung cancer sufferers.

\section{References}

1. K. Mori, Y. Saito, K. Tominaga, K. Yokoi, N. Miyazawa, A. Okuyama, and M. Sasagawa. Small nodular lesions in the lung periphery: New approach to diagnosis with CT. Radiology, 177:843-849, 1990.

2. Y. Kawata, N. Niki, and H. Ohmatsu. Curvature-based Internal Structure Analysis of Pulmonary Nodules Using Thoracic 3D CT Images. Systems and Computers in Japan, 32(11), 2001.

3. T. Masami, E. Masahiro, N. Koji, K. Masayuki, Y. Satory, K. Kiyomitsu, and H. Kazuyuki. Conceptual design of a synchrotron light source used for medical diagnoses at NIRS. In SPIE, volume 3770, pages 213-220, 1999.

4. Chiou YSP, Lure YMF, Ligomenides PA. Neural Network Image Analysis and Classification in Hybrid Lung Nodule Detection (HLND) System. In IEEE-SP Workshop on Neural Networks for Signal Processing, pages 517-526, 1993.

5. T. Tanaka, K. Yuta and Y. Kobayashi. A Study of False-Negative Case in MassScreening of Lung Cancer. Jap. Thor. Med., 43:832-838, 1984.

6. J. Oda, S. Akita and K. Shimada. A Study of False-Negative Case in Comparative Reading of Mass-Screening of Lung Cancer. Lung Cancer, 29:271-278, 1989.

7. M. Koizumi. Medical Image Analysis for the Diagnosis of Chest X-ray. Doctor of Engineering Dissertation, Graduate School of Engineering, Osaka City University, Osaka, Japan, 2003. 37-47.

8. K. Kanazawa, M. Kubo and N. Niki. Computer Aided Diagnosis System for Lung Cancer Based on Helical CT Images. In 13th International Conference on Pattern Recognition, volume 3, pages 381-385, 1996.

9. A.K. Jain. Fundamentals of Digital Image Processing. Prentice-Hall Inc., 1989.

10. Mokhtarian F and Machworth. A Scale-Based Description of Planner Curves and Two-Dimensional Shapes. IEEE Trans. on Pattern Analysis and Matching Intelligence, 8:34-43, 1996. 